\title{
Epiphytic mosses from the genera Orthotrichum s.l. and Nyholmiella in the Nízký Jeseník Mts (Northern Moravia, Czech Republic).
}

\author{
Lukáš Číhal, Oto Kaláb \& Michaela Mrnková
}

\begin{abstract}
Epiphytic mosses from the genera Orthotrichum s.l. and Nyholmiella in the Nízký Jeseník Mts (Northern Moravia, Czech Republic ). - Acta Mus. Siles. Sci. Natur., 64: 207-213, 2015.

Abstract: The study presents the distribution of the genera Orthotrichum s.l. and Nyholmiella in the phytogeographical district 98. Nízký Jeseník, and follows the project carried out between years 2005 and 2009 in nearby phytogeographical district 97 . Hrubý Jeseník. The total number of 57 locations was visited during the year 2015, and 142 samples of mosses were collected. Altogether, 9 species of genus Orthotrichum s.l. and 1 species of the genus Nyholmiella were recently recorded in the studied area. Lists of species and localities are given.
\end{abstract}

Key words: Bryofloristic research, Orthotrichum s.l., Nyholmiella, Nízký Jeseník Mts, Hrubý Jeseník Mts

\section{Introduction}

The genus Orthotrichum s.l. comprises of at least 164 species (Medina et al. 2013, updated according to Plášek et al. 2014) and the genus Nyholmiella includes two taxa (Sawicki et al. 2010). They are mainly epiphytic, less often epilithic mosses (Plášek 2012), which are widespread throughout the world from the Arctic to the Antarctic, except in deserts and wet tropical forests (Lewinsky 1993).

The study was carried out in the phytogeographical district Nízký Jeseník, the flat highlands of Northern Moravia and south part of Silesia (Czech Republic) and also one of the oldest geological units in Central Europe with the remains of volcanic activity. The geomorphological units occurring in the district belong to: IVC Jesenická subset, IVC-8 Nízký Jeseník, IVC-8C Bruntálská vrchovina highlands, IVC-8D Slunečná vrchovina highlands and IVC-8E Domašovská vrchovina highlands (Bína et al. 2012). According to the "climatic classification" (Quitt 1971), the region of Nízký Jeseník belongs mainly to mildly warm areas with the exception of the Slunečná hora Mt., which belongs to a cold area. Snow cover is usually maintained only for a short part of the year.

Both districts are classified as Czech oreophyticum (Oreophyticum Massivi bohemici), mountainous areas with prevailing cold-flora (Skalický 1988). Hrubý Jeseník was formed by the eastern section of the Sudeten mountain system and is situated more in the north of Moravia (Czech Republic). The average height is about $890 \mathrm{~m}$ a.s.l., and the highest point is Praděd Mt. (1491 m a.s.1.). The average height in Nízký Jeseník is $450 \mathrm{~m}$ a.s.l., and the highest point is Slunečná hora Mt. (800 m a.s.1.), therefore, we can also assume differences in ecological characteristics.

The differences between the districts are not only in their ecological characteristics, but also in their size. The phytogeographical district Hrubý Jeseník with its area of 61603 ha is more than three and a half times bigger than the Nízký Jeseník district with 17205 ha. In their land cover, there are differences especially in the presence of coniferous forests and meadows and pastures. For land cover comparsion, CORINE Land Cover 2012 data were analysed using QGIS (QGIS Development Team 2015). The most common type of vegetation in both districts was coniferous forest (first mentioned are always the data for Hrubý Jeseník and 
second for Nízký Jesenik), 40596 ha, 65.9\% (percentual cover of the district); 6621 ha, $38.49 \%$, in which the species of genus Orthotrichum grow only sporadically. They can be expected more often in the mixed forest, 7231 ha, $11.74 \% ; 1526$ ha and $8.87 \%$, and also in the leaf forest, $1607 \mathrm{ha}, 2.61 \%$; $111 \mathrm{ha}, 0.65 \%$. Other important types of vegetation were meadows and pastures, 2393 ha, 3.89\%; 6812 ha, 39.59\%, much more present in Nízký Jeseník, and low vegetation in the forest with 5347 ha, $8.68 \%$ and 504 ha, 2.93\%. The last important type of vegetation were agricultural areas with natural vegetation, 2929 ha, 4.76\%; 958 ha, $5.57 \%$.

\section{Methods}

The research was carried out in 2015 and includes excerption of literature, herbarium data and field survey. For data comparison were also used records coming from the bryological database of V. Plášek. Recent material was collected by the first author in the locations listed below. For comparison with the previous list of locations and species collected from Hrubý Jeseník Mts see Č́íhal et al. (2012). GPS positions of historical data were added. All recent specimens were collected and identified by the first author. The items are housed in OP.

\section{A list of localities}

\section{Recent localities:}

$10.47 \mathrm{~km}$ W of Stará Libavá village, $2.4 \mathrm{~km}$ SE of Moravský Beroun town; $578 \mathrm{~m}$ a.s.l.; WGS-84: $49^{\circ} 46^{\prime} 52.1893 " \mathrm{~N} 17^{\circ} 29^{\prime} 07.1844 " \mathrm{E}$

$20.48 \mathrm{~km} \mathrm{NW}$ of Moravský Beroun town, $1.63 \mathrm{~km}$ NE from centre of Ondrášov village, along public road; 592 m a.s.l.; WGS-84: $49^{\circ} 48^{\prime} 16.2119^{\prime \prime} \mathrm{N} 17^{\circ} 26^{\prime} 10.0860^{\prime \prime} \mathrm{E}$

$3 \quad 0.51 \mathrm{~km}$ NW of Moravský Beroun town, $1.65 \mathrm{~km} \mathrm{NE}$ from centre of Ondrášov village, along public road; 582 m a.s.l.; WGS-84: $49^{\circ} 48^{\prime} 18.5918^{\prime \prime} \mathrm{N} 17^{\circ} 26^{\prime} 09.7512^{\prime \prime} \mathrm{E}$

$40.63 \mathrm{~km}$ NW of Moravský Beroun town, $3 \mathrm{~km}$ SE of Dětřichov nad Bystřicí village, along public road; 599

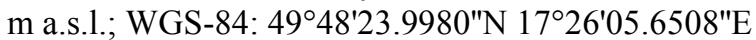

$5 \quad 0.66$ km SSE of Dětrichov nad Bystřicí village; 599 m a.s.1.; WGS-84: 4948'38.4998"N 17²3'49.0992"E

$60.8 \mathrm{~km}$ NW of Moravský Beroun town, $3.7 \mathrm{~km}$ SE of Dětřichov nad Bystřicí village, along public road; 608 m a.s.l.; WGS-84: $49^{\circ} 48^{\prime} 15.3000^{\prime \prime} \mathrm{N} 17^{\circ} 26^{\prime} 10.6008^{\prime \prime} \mathrm{E}$

$7 \quad 0.82 \mathrm{~km} \mathrm{NW}$ of Dětrichov nad Bystřicí village, $2.97 \mathrm{~km} \mathrm{NW}$ of Moravský Beroun town; $587 \mathrm{~m}$ a.s.l.; WGS-84: 4948'39.0499"N 17²4'06.1596"E

$81 \mathrm{~km} \mathrm{E}$ of Dalov village, 1.6 km NW of Horní Loděnice village; 588 m a.s.1.; WGS-84: 4946'59.2799"N $17^{\circ} 21^{\prime} 12.2508^{\prime \prime} \mathrm{E}$

$9 \quad 1.02 \mathrm{~km} \mathrm{NW}$ of Moravský Beroun town, $2.04 \mathrm{~km}$ NE from centre of Ondrášov village, along public road; 601 m a.s.l.; WGS-84: $49^{\circ} 48^{\prime} 21.6000^{\prime \prime} \mathrm{N} 17^{\circ} 26^{\prime} 07.6992^{\prime \prime} \mathrm{E}$

$101.25 \mathrm{~km} \mathrm{NW}$ of Moravský Beroun town, $0.9 \mathrm{~km}$ od north edge of Ondrášov village, along railway; $573 \mathrm{~m}$ a.s.l.; WGS-84: $49^{\circ} 48^{\prime} 04.2001^{\prime \prime N} 17^{\circ} 25^{\prime} 22.0008^{\prime \prime} \mathrm{E}$

$111.26 \mathrm{~km}$ NNE of Moravský Beroun town, $2.86 \mathrm{~km} \mathrm{NE}$ from centre of Ondrášov village, along public road; 588 m a.s.l.; WGS-84: $49^{\circ} 48^{\prime} 33.9001^{\prime \prime N} 17^{\circ} 26^{\prime} 56.1984 " \mathrm{E}$

$121.3 \mathrm{~km}$ of Moravský Beroun Town, $3.35 \mathrm{~km}$ SE of Dětřichov nad Bystřicí village, along public road; $586 \mathrm{~m}$ a.s.l.; WGS-84: $49^{\circ} 48^{\prime} 31.3999^{\prime \prime} \mathrm{N} 17^{\circ} 25^{\prime} 59.5992$ "E

$131.33 \mathrm{~km}$ SSE of Čabová village, $1.61 \mathrm{~km} \mathrm{E}$ of Moravský Beroun town; $580 \mathrm{~m}$ a.s.l.; WGS-84: $49^{\circ} 47^{\prime} 54.5424 " \mathrm{~N} 17^{\circ} 29^{\prime} 16.5552 \mathrm{E}$

$141.57 \mathrm{~km}$ NE of Nová Véska village, $0.4 \mathrm{~km} \mathrm{~W}$ of Stará Libavá village; $583 \mathrm{~m}$ a.s.l.; WGS-84: $49^{\circ} 46^{\prime} 51.7343^{\prime \prime} \mathrm{N} 17^{\circ} 29^{\prime} 26.0088^{\prime \prime} \mathrm{E}$

$151.6 \mathrm{~km} \mathrm{NW}$ of Nové Valteřice village, touristic path to Slunečná hora Mt; $697 \mathrm{~m}$ a.s.1.; WGS-84: $49^{\circ} 50^{\prime} 59.5021^{\prime \prime} \mathrm{N} 17^{\circ} 26^{\prime} 55.4388^{\prime \prime} \mathrm{E}$

$161.60 \mathrm{E}$ of Moravský Beroun, $1.25 \mathrm{~km}$ SSE od Čabová village; $603 \mathrm{~m}$ a.s.l.; WGS-84: 4947'54.5424"N $17^{\circ} 29^{\prime} 16.5552^{\prime \prime} \mathrm{E}$

$171.61 \mathrm{~km} \mathrm{NE}$ of Nová Véska village, $0.15 \mathrm{~km} \mathrm{~W}$ of Stará Libavá village; $609 \mathrm{~m}$ a.s.l.; WGS-84: $49^{\circ} 46^{\prime} 51.8837^{\prime \prime} \mathrm{N} 17^{\circ} 29^{\prime} 41.4780^{\prime \prime} \mathrm{E}$

$181.62 \mathrm{~km} \mathrm{E}$ of Moravský Beroun town, $1.33 \mathrm{~km}$ SSE of Čabová village; $577 \mathrm{~m}$ a.s.l.; WGS-84: $49^{\circ} 47^{\prime} 55.0424^{\prime \prime} \mathrm{N} 17^{\circ} 29^{\prime} 20.4540 " \mathrm{E}$

$191.74 \mathrm{~km}$ E of Dětrichov nad Bystřicí village, $4.36 \mathrm{~km}$ NNW of Moravský Beroun town; $581 \mathrm{~m}$ a.s.l.; WGS-

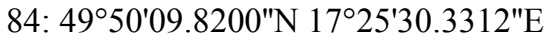

$201.8 \mathrm{~km}$ N o Moravský Beroun town, $3 \mathrm{~km}$ SE of Dětrrichov nad Bystřicí village, along pasture; $585 \mathrm{~m}$ a.s.l.; WGS-84: $49^{\circ} 48^{\prime} 42.9098^{\prime \prime} \mathrm{N} 17^{\circ} 26^{\prime} 02.0004^{\prime \prime} \mathrm{E}$ 
$211.8 \mathrm{~km}$ NW of Moravský Beroun town, $3.8 \mathrm{~km}$ SE of Dětřichov nad Bystřicí village, along public road; 567 m a.s.l.; WGS-84: 4948'52.3001"N 17²6'58.8984"E

$221.83 \mathrm{~km} \mathrm{~N}$ of Moravský Beroun town, along guesthouse Gizita, along public road; $567 \mathrm{~m}$ a.s.l.; WGS-84:

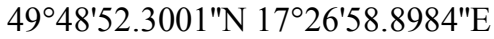

$231.84 \mathrm{~km} \mathrm{~N}$ of Moravský Beroun town, $1.48 \mathrm{~km} \mathrm{NW}$ of Čabová village, along public road; $592 \mathrm{~m}$ a.s.1.; WGS-84: 4948'52.8001"N 17²6'59.3988"E

$241.89 \mathrm{~km} \mathrm{~N}$ of Moravský Broun town, $0.2 \mathrm{~km} \mathrm{SW}$ of Gizita guesthouse, along public road; $566 \mathrm{~m}$ a.s.1.; WGS-84: 4948'52.8001"N 17²6'59.3988"E

$252 \mathrm{~km}$ WNW of Krrišt’anovice village, $0.72 \mathrm{~km}$ NNE of Nové Valterice village, "Důlní potok" stream; $663 \mathrm{~m}$ a.s.l.; WGS-84: 4951'19.2499"N 17²8'50.9016"E

$262.24 \mathrm{~km} \mathrm{~N}$ of Moravský Broun town, $0.35 \mathrm{~km} \mathrm{NW}$ of Gizita guesthouse, along public road; $577 \mathrm{~m}$ a.s.1.; WGS-84: 4949'04.2571"N 17²6'54.1248"E

$272.3 \mathrm{~km} \mathrm{NW}$ of Čabová village, $2.52 \mathrm{~km} \mathrm{~N}$ of Moravský Beroun town, on solitere pasture; $601 \mathrm{~m}$ a.s.1.; WGS-84: 49॰49'15.3998"N 17²6'39.0012"E

$282.3 \mathrm{~km}$ SEE of Dětřichov nad Bystřicí village, $3.25 \mathrm{~km} \mathrm{~N}$ of Moravský Beroun town, along touristic path; 710 m a.s.1.; WGS-84: 4949'34.6321"N 17²5'55.1856"E

$292.46 \mathrm{~km}$ SE of Lomnice village, $2.49 \mathrm{~km}$ NW of Nové Valteřice village, loc. "Vysoký kámen", $2 \mathrm{~km}$ from top "Slunečná hora Mt.", along the red tourist trail; $743 \mathrm{~m}$ a.s.1.; WGS-84: 4951'15.9800"N $17^{\circ} 26^{\prime} 19.7088^{\prime \prime E}$

$302.48 \mathrm{~km} \mathrm{~N}$ of Moravský Beroun town, $3.7 \mathrm{~km}$ E of Dětrichov nad Bystřicí village; $595 \mathrm{~m}$ a.s.1.; WGS-84: $49^{\circ} 49^{\prime} 15.1298^{\prime \prime} \mathrm{N} 17^{\circ} 26^{\prime} 57.0300^{\prime \prime} \mathrm{E}$

$312.6 \mathrm{~km}$ NW of Nové Valteřice village, touristic path to Slunečná hora Mt., loc. "Vysoký kámen"; $746 \mathrm{~m}$ a.s.l.; WGS-84: 4951'15.0419"N 17²6'18.0492"E

$32 \quad 2.63 \mathrm{~km} \mathrm{~N}$ from Moravský Beroun town, $3.9 \mathrm{~km}$ E from Dětrichov nad Bystřicí village, dir. Nové Valteřice village; 567 m a.s.1.; WGS-84: 4949'18.7388"N 17²6'59.8992"E

$332.7 \mathrm{~km}$ N of Moravský Beroun town, $3.84 \mathrm{~km}$ SE from Dětrichov nad Bystřicí, along public road; $607 \mathrm{~m}$ a.s.1.; WGS-84: 4948'57.1000"N 17²6'56.1012"E

$342.81 \mathrm{~km} \mathrm{~N}$ of Moravský Beroun town, $3.63 \mathrm{~km}$ E from Dětrichov nad Bystřicí, on solitere pasture; $597 \mathrm{~m}$ a.s.l.; WGS-84: 4949'24.7001"N 17²6'44.4984"E

$353 \mathrm{~km} \mathrm{~N}$ of Moravský Beroun town, edge of pasture; $614 \mathrm{~m}$ a.s.1.; WGS-84: 4949'31.0001"N $17^{\circ} 26^{\prime} 46.5000^{\prime \prime} \mathrm{E}$

$363.93 \mathrm{~km}$ ESE of Dětrichov nad Bystřicí village, $2.96 \mathrm{~km}$ NNE of Moravský Beroun town, dir. Nové Valteřice village; $626 \mathrm{~m}$ a.s.1.; WGS-84: 4949'28.8502"N 17²2'14.0688"E

$374.06 \mathrm{~km}$ E of Dětrichov nad Bystřicí village, $2.76 \mathrm{~km} \mathrm{~N}$ of Moravský Beroun town; 578 m a.s.1.; WGS-84: $49^{\circ} 49^{\prime} 21.5699^{\prime \prime} \mathrm{N} 17^{\circ} 27^{\prime} 16.3008^{\prime \prime} \mathrm{E}$

$384.37 \mathrm{~km}$ E of Dětrichov nad Bystřicí village, $3.55 \mathrm{~km}$ NNE of Moravský Beroun town; $581 \mathrm{~m}$ a.s.l.; WGS84: 4949'43.1299"N 17²7'28.9008"E

$394.39 \mathrm{SE}$ of Lomnice village, $2.63 \mathrm{~km} \mathrm{~W}$ of Křišt'anovice village, at crossroad; $680 \mathrm{~m}$ a.s.l.; WGS-84: $49^{\circ} 51^{\prime} 22.9298^{\prime \prime} \mathrm{N} 17^{\circ} 28^{\prime} 21.6084^{\prime \prime} \mathrm{E}$

40 Centre of Lomnice village, $10 \mathrm{~m}$ from small bridge; $651 \mathrm{~m}$ a.s.1.; WGS-84: 4952'20.7001"N $17^{\circ} 25^{\prime} 06.0996 " \mathrm{E}$

41 Centre of Lomnice village, along railway; $593 \mathrm{~m}$ a.s.1.; WGS-84: 4952'20.7001"N 17²5'06.2004"E

42 Centre of Lomnice village, along stream; $591 \mathrm{~m}$ a.s.1.; WGS-84: 4952'17.8000"N 17²4'58.2984"E

43 Centre of Lomnice village, along stream; $601 \mathrm{~m}$ a.s.1.; WGS-84: 4952'22.3000"N 17²5'01.5.996"E

44 Centre of Lomnice village, along stream; $584 \mathrm{~m}$ a.s.1.; WGS-84: 4952'26.5001"N 17²5'04.4004"E

45 Centre of Lomnice village, next to bridge along stream; $649 \mathrm{~m}$ a.s.l.; WGS-84: 4952'26.5001"N $17^{\circ} 25^{\prime} 04.4004 " \mathrm{E}$

46 Centre of Lomnice village, the path to the cemetery; $605 \mathrm{~m}$ a.s.1.; WGS-84: 4952'26.2999"N $17^{\circ} 24^{\prime} 58.5000 " \mathrm{E}$

47 Centre of Norberčany village; 568 m a.s.1.; WGS-84: 4945'03.6799"N 17³0'37.5696"E

48 Centre of Nové Valteřice village; $610 \mathrm{~m}$ a.s.1.; WGS-84: 4950'38.0198"N 17²8'22.7316"E

49 E edge of the Lomnice village, next to bridge; $593 \mathrm{~m}$ a.s.1.; WGS-84: 4952'20.7001"N 17²25'06.2004"E

50 E edge of the Lomnice village, under the bridge, along railway; $610 \mathrm{~m}$ a.s.1.; WGS-84: 4952'20.7001"N $17^{\circ} 25^{\prime} 06.2004^{\prime \prime} \mathrm{E}$

$51 \mathrm{~N}$ edge of Moravský Beroun town, near public road; $599 \mathrm{~m}$ a.s.l.; WGS-84: 4948'12.2350"N 17²6'15.9432"E

52 N edge of Norberčany village; 603 m a.s.1.; WGS-84: 4945'22.5900"N 17³0'13.7484"E

53 N edge of Ondrášov village, along public road; $545 \mathrm{~m}$ a.s.1.; WGS-84: 4947'46.8067"N 17²5'27.4152"E

$54 \mathrm{~N}$ part of Ondrášov village, $0.8 \mathrm{~km}$ W of Moravský Beroun town, along railway; $549 \mathrm{~m}$ a.s.1.; WGS-84: $49^{\circ} 47^{\prime} 54.5186^{\prime \prime} \mathrm{N} 17^{\circ} 25^{\prime} 18.4800^{\prime \prime} \mathrm{E}$ 
55 Nové Valteřice village, close to the church, along public road; $212 \mathrm{~m}$ a.s.l.; WGS-84: 4950'27.4369"N $17^{\circ} 28 ' 22.7856 " \mathrm{E}$

56 NW edge of Moravský Beroun; 600 m a.s.l.; WGS-84: 4948'12.0348"N 17²6'15.9432"E

57 S edge of Dětrichov nad Bystřicí village; 599 m a.s.1.; WGS-84: 49²4'55.1498"N 17²3'36.3588"E

\section{Historical localities:}

$581.5 \mathrm{~km}$ E of Lomnice u Rýmařova village, kamenec hill along public road.; $700 \mathrm{~m}$ a.s.l.; WGS-84: $49^{\circ} 52^{\prime} 37.2700^{\prime \prime} \mathrm{N} 17^{\circ} 26^{\prime} 31.3200 " \mathrm{E}$

59 Moravský Beroun town, $1.2 \mathrm{~km} \mathrm{NNW}$ of Nové Valteřice village, near a public road.; $640 \mathrm{~m}$ a.s.l.; WGS84: $49^{\circ} 51^{\prime} 10.2000^{\prime \prime} \mathrm{N} 17^{\circ} 28^{\prime} 19.8500^{\prime \prime} \mathrm{E}$

60 Nové Valteřice village, $5.2 \mathrm{~km}$ of Moravský Beroun town.; $640 \mathrm{~m}$ a.s.l.; WGS-84: 4950'32.4200"N $17^{\circ} 28^{\prime} 22.2400^{\prime \prime} \mathrm{E}$

\section{Results}

\section{A list of recorded species}

The taxa are sorted in alphabetical order. Numbers of locations are written in bold font and are sorted in ascending order. The list is divided into two parts, recent and historical data. The phorophyte species from which the moss specimens were collected are written in italic font. Sources of literature data are in brackets. All recent data were collected and identified by the first author and are housed in herbarium of Silesian museum in Opava (OP).

The nomenclature of bryophytes follows Kučera, Váňa \& Hradílek (2012). Each species placed on the list contains threat status according to Kučera, Váňa \& Hradílek (2012), which is presented in brackets after its Latin name.

\section{Nyholmiella obtusifolia (Brid.) Holmen et E. Warncke [LC]:}

21 (07. VII. 2015; Sorbus aucuparia); 37 (08. VII. 2015; Fraxinus excelsior); 48 (08. VII. 2015; Fraxinus excelsior).

\section{Orthotrichum affine Schrad. ex Brid. var. affine [LC]:}

1 (06. V. 2015; Fraxinus excelsior); 9 (07. VII. 2015; Fraxinus excelsior); 12 (07. VII. 2015; Fraxinus excelsior); 14 (06. V. 2015; Fraxinus excelsior); 15 (08. VII. 2015; Acer sp.); 56 (09. VII. 2015; Tilia cordata) 59 (26. VIII. 1974; Ulmus glabra; leg. V. Pospíšil; det. Vondráček; BRNM; [Vondráček 1993]); 60 (22. VI. 1983; Acer sp.; leg. I. Novotný; BRNM; [Vondráček 1993]).

\section{Orthotrichum anomalum Hedw. [LC]:}

12 (07. VII. 2015; Fraxinus excelsior); 32 (10. VII. 2015; Tilia cordata); 41 (21. V. 2015; Fraxinus excelsior); 42 (21. V. 2015; Acer sp.); 42 (21. V. 2015; Sambucus nigra); 44 (21. V. 2015; Salix sp., Fraxinus excelsior); 50 (22. V. 2015; Fraxinus excelsior); 53 (09. VII. 2015; Acer sp.).

\section{Orthotrichum diaphanum Schrad. ex Brid. [LC]:}

1 (06. V. 2015; Fraxinus excelsior); 6 (06. VII. 2015; Fraxinus excelsior); 8 (25. X. 2015; Fagus sylvatica); 9 (07. VII. 2015; Fraxinus excelsior); 12 (07. VII. 2015; Fraxinus excelsior); 14 (06. V. 2015; Fraxinus excelsior); 38 (08. VII. 2015; Acer sp.); 41 (21. V. 2015; Fraxinus excelsior); 45 (22. V. 2015; Acer sp.); 46 (22. V. 2015; Fraxinus excelsior); 56 (09. VII. 2015; Tilia cordata).

\section{Orthotrichum pallens Bruch ex Brid. [LC]:}

3 (06. VII. 2015; Fraxinus excelsior); 4 (06. VII. 2015; Acer sp.); 5 (24. X. 2015; Salix sp.); 6 (06. VII. 2015; Fraxinus excelsior); 7 (24. X. 2015; Salix sp.); 8 (25. X. 2015; Fagus sylvatica); 9 (07. VII. 2015; Fraxinus excelsior); 11 (09. VII. 2015; Salix sp.); 12 (07. VII. 2015; Fraxinus excelsior); 16 (06. V. 2015; Alnus glutinosa); 20 (07. VII. 2015; Fraxinus excelsior); 21 (07. VII. 2015; Sorbus aucuparia); 22 (09. VII. 2015; Tilia cordata); 23 (09. VII. 2015; Acer sp.); 25 (24. X. 2015; Fagus sylvatica); 26 (08. VII. 2015; Fraxinus excelsior); 27 (07. VII. 2015; Crataegus sp.); 28 (08. VII. 2015; Acer sp.); 29 (08. VII. 2015; Acer sp.); 30 (10. VII. 2015; Fraxinus excelsior); 31 (08. VII. 2015; Fagus sylvatica); 32 (10. VII. 2015; Tilia cordata); 33 (10. VII. 2015; 
Salix sp.); 35 (07. VII. 2015; Betula pendula); 36 (08. VII. 2015; Fraxinus excelsior); 38 (08. VII. 2015; Acer sp.); 39 (24. X. 2015; Acer sp.); 44 (21. V. 2015; Salix sp., Fraxinus excelsior); 46 (22. V. 2015; Fraxinus excelsior); 48 (08. VII. 2015; Fraxinus excelsior); 53 (09. VII. 2015; Acer sp.); 57 (25. X. 2015; Salix sp.).

Orthotrichum patens Bruch ex Brid. LR-nt

18 (06. V. 2015; Carpinus betulus).

Orthotrichum pumilum Sw. ex Anon. [LC]:

1 (06. V. 2015; Fraxinus excelsior); 2 (06. VII. 2015; Acer sp.); 4 (06. VII. 2015; Acer sp.); 6 (06. VII. 2015; Fraxinus excelsior); 9 (07. VII. 2015; Fraxinus excelsior); 10 (09. VII. 2015; Salix sp.); 14 (06. V. 2015; Fraxinus excelsior); 15 (08. VII. 2015; Acer sp.); 17 (06. V. 2015; Fraxinus excelsior); 21 (07. VII. 2015; Sorbus aucuparia); 23 (09. VII. 2015; Acer sp.); 24 (09. VII. 2015; Fraxinus excelsior); 36 (08. VII. 2015; Fraxinus excelsior); 37 (08. VII. 2015; Fraxinus excelsior); 40 (22. V. 2015; Acer sp.); 41 (21. V. 2015; Fraxinus excelsior); 42 (21. V. 2015; Acer sp., Sambucus nigra); 43 (21. V. 2015; Acer sp.); 44 (21. V. 2015; Salix sp.); 45 (22. V. 2015; Acer sp.); 46 (22. V. 2015; Fraxinus excelsior); 49 (22. V. 2015; Tilia cordata); 51 (09. VII. 2015; Acer sp.); 54 (09. VII. 2015; Salix sp.); 55 (08. VII. 2015; Acer sp.).

\section{Orthotrichum speciosum Nees [LC]:}

1 (06. V. 2015; Fraxinus excelsior); 3 (06. VII. 2015; Fraxinus excelsior); 4 (06. VII. 2015; Acer sp.); 6 (06. VII. 2015; Fraxinus excelsior); 7 (24. X. 2015; Salix sp.); 8 (25. X. 2015; Fagus sylvatica); 9 (07. VII. 2015; Fraxinus excelsior); 10 (09. VII. 2015; Salix sp.); 13 (06. V. 2015; Salix sp.); 14 (06. V. 2015; Fraxinus excelsior); 15 (08. VII. 2015; Acer sp.); 16 (06. V. 2015; Alnus glutinosa); 17 (06. V. 2015; Fraxinus excelsior); 19 (08. VII. 2015; Fraxinus excelsior); 20 (07. VII. 2015; Fraxinus excelsior); 21 (07. VII. 2015; Sorbus aucuparia); 23 (09. VII. 2015; Acer sp.); 24 (09. VII. 2015; Fraxinus excelsior); 25 (24. X. 2015; Fagus sylvatica); 26 (08. VII. 2015; Fraxinus excelsior); 28 (08. VII. 2015; Acer sp.); 29 (08. VII. 2015; Acer sp.); 30 (10. VII. 2015; Fraxinus excelsior); 32 (10. VII. 2015; Tilia cordata); 33 (10. VII. 2015; Salix sp.); 34 (10. VII. 2015; Alnus glutinosa); 35 (07. VII. 2015; Betula pendula); 36 (08. VII. 2015; Fraxinus excelsior); 38 (08. VII. 2015; Acer sp.); 39 (24. X. 2015; Acer sp.); 41 (21. V. 2015; Fraxinus excelsior); 42 (21. V. 2015; Acer sp., Sambucus nigra); 44 (21. V. 2015; Salix sp., Fraxinus excelsior); 46 (22. V. 2015; Fraxinus excelsior); 47 (25. X. 2015; Acer sp.); 48 (08. VII. 2015; Fraxinus excelsior); 51 (09. VII. 2015; Acer sp.); 52 (25. X. 2015; Salix sp.); 53 (09. VII. 2015; Acer sp.); 54 (09. VII. 2015; Salix sp.); 55 (08. VII. 2015; Acer sp.); 56 (09. VII. 2015; Tilia cordata); 57 (25. X. 2015; Salix sp.).

Orthotrichum stramineum Hornsch. ex Brid. [LC]:

18 (06. V. 2015; Carpinus betulus); 30 (10. VII. 2015; Fraxinus excelsior); 44 (21. V. 2015; Fraxinus excelsior) 59 (16. VIII. 1960; Sorbus aucuparia; leg. J. Stuchlý; det. Vondráček; PRC; [Vondráček 1993]).

Orthotrichum striatum Hedw. [LC-att]:

6 (06. VII. 2015; Fraxinus excelsior); 8 (25. X. 2015; Fagus sylvatica); 17 (06. V. 2015; Fraxinus excelsior); 28 (08. VII. 2015; Acer sp.); 31 (08. VII. 2015; Fagus sylvatica).

\section{Discussion}

The area of the phytogeographical district Nízký Jeseník has never been purposefully subjected to any bryological research focused on genera Orthotrichum s.1. and Nyholmiella, so the historical data for Nízký Jeseník are almost unknown (3 samples). For comparision, from Hrubý Jeseník there are 48 historical and 197 recent samples. Also lower is the number of recently collected samples from Nízký Jeseník (142 samples). Even through these differences, the number of recently recorded species is same in both districts, altogether, 9 species of genus Orthotrichum s.l. and 1 species of the genus Nyholmiella. The same species for both districts are: Nyholmiella obtusifolia, Orthotrichum affine, $O$. anomalum, $O$. diaphanum, $O$. pallens, $O$. pumilum, $O$. speciosum, $O$. stramineum, and $O$. striatum. The species $O$. patens is recently known only from Nízký Jeseník and $O$. alpestre is recently known only from Hrubý 
Jeseník. From Hrubý Jeseník is also known one species which doesn’t occur recently in both districts, O. rupestre found in two localities by Duda (1950).

The biggest difference in number of samples altogether is in species $O$. stramineum, 45/4, $O$. affine, $38 / 8$ and $N$. obtusifolia, 18/3, while the lower numbers are for Nízký Jeseník. Only for $O$. pallens, $25 / 33$, is the number of samples higher for Nízký Jeseník and for $O$. anomalum, the number is the same, 9/9. Among the most abundant species in both areas, O. speciosum (48/45) could be mentioned.

Among the most interesting recently recorded species from Nízký Jeseník, O. patens can be mentioned. Earlier, the moss was considered as rare, but the ongoing climate change has a beneficial effect on the spreading of this species and the number of locations is increasing, not only in Czech Republic, but in the whole Central Europe (Plášek et al. 2012).

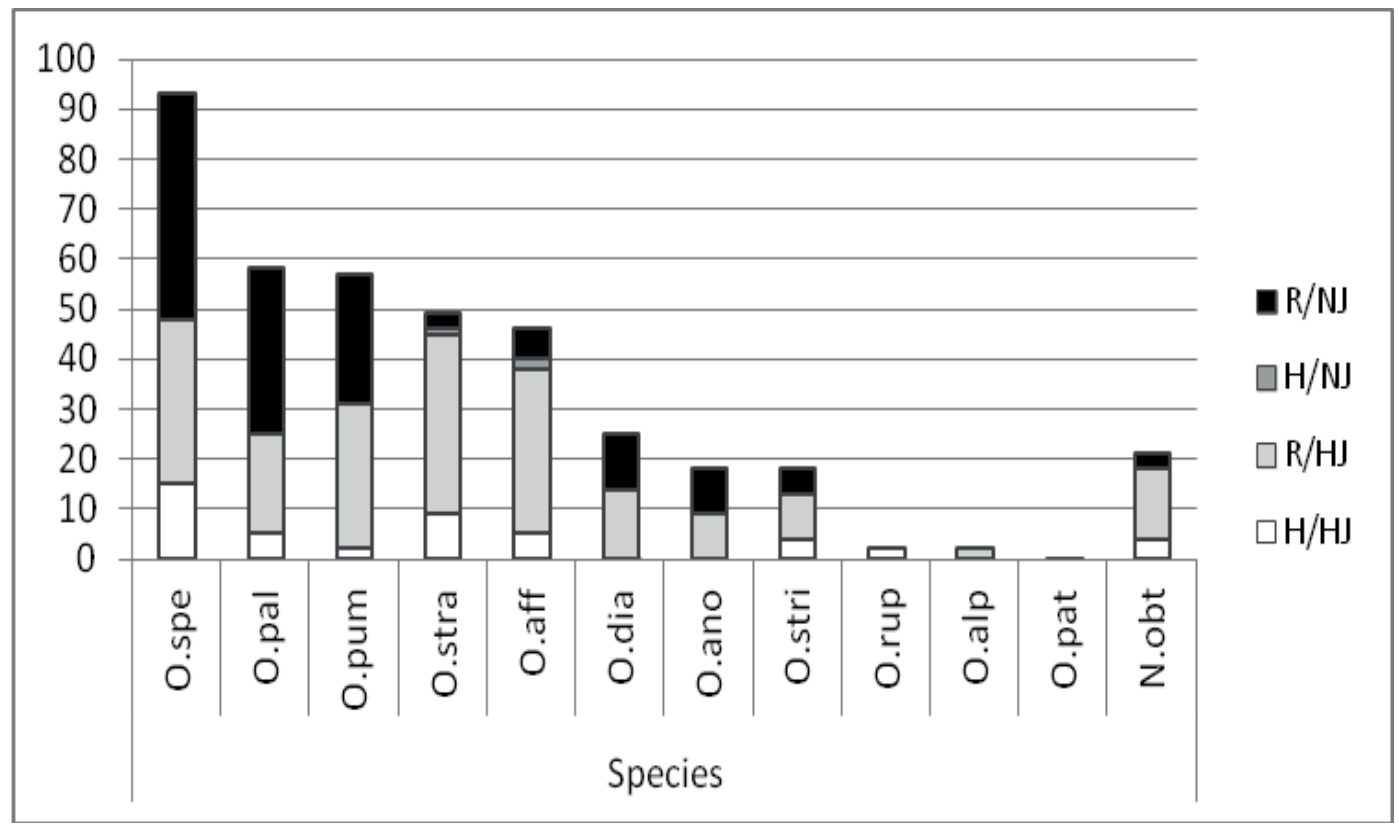

Fig 1: Summarization of historical and recent sampling effort of epiphytic mosses in the area of Hrubý and Nízký Jeseník phytogeographical districts. Shortcuts used in the graph are: recently recorded samples from Nízký Jeseník (R/NJ), historical data from Nízký Jeseník H/NJ, recently recorded samples from Hrubý Jeseník (R/HJ) and historical samples from Hrubý Jeseník (H/HJ). Species from the left: Orthotrichum speciosum, O. pallens, O. pumilum, O. stramineum, O. affine, O. diaphanum, O. anomalum, O. striatum, O. rupestre, O. alpestre, O. patens, Nyholmiella obtusifolia.

Acknowledgement: The study was financially supported by the Ministry of Culture of the Czech Republic by institutional financing of long-term conceptual development of the research institution (the Silesian Museum, MK000100595), internal grant of the Silesian Museum No. IGS201501/2015.

\section{References}

Bína J. \& Demek J. (2012): Z nížin do hor: geomorfologické jednotky České republiky. Academia, 344 pp.

Č́nal L. \& Plášek V. (2012): Critical evaluation of epiphytic mosses from Orthotrichaceae family in the Hrubý Jeseník Mts (Northern Moravia, Czech Republic). - C̆as. Slez. Muz. Opava (A) 61: 113-122.

Duda (1950): Výsledek bryologického výzkumu Slezska. I. - Př́rod. Sborn. Ostrav. Kraje, Opava 11: 327-337.

Kučera J., Váňa J. \& Hradílek Z. (2012): Bryophyte flora of the Czech Republic: updated checklist and Red List and a brief analysis. - Preslia 84: 813-850.

Lewinsky J. (1993): A synopsis of the genus Orthotrichum Hedw. (Musci, Orthotrichaceae). - Bryobrothera 2: 1-59. 
Medina R., Lara F., Goffinet B., Garilleti R. \& Mazimpaka V. (2013): Unnoticed diversity within the disjunct moss Orthotrichum tenellum s.l. validated by morphological and molecular approaches.Taxon 62(6): 1133-1152.

Plášek V., Sawicki J. \& Č́hal L. (2014): Orthotrichum pamiricum (Bryophyta), a new epiphytic moss species from Pamir Mountains in Central Asia. - Turkish Journal of Botany 38(4) : 754-762.

QGIS Development Team (2015): QGIS Geographic Information System. Open Source Geospatial Foundation Project. http://qgis.osgeo.org.

Quitt E. (1971): Klimatické oblasti Československa, Academia, 73 pp.

Sawicki J., Plášek V. \& Szczecińska M. (2010): Molecular studies resolve Nyholmiella (Orthotrichaceae) as a separate genus. - Journal of Systematics and Evolution 48: 183-194.

Skalický V. (1988): Regionálně fytogeografické členění. In: Hejný S. \& Slavík B.: Květena ČSR I., Academia, pp. 103-121.

Vondráček M. (1993): Revize a rozšíření druhů rodu Orthotrichum Hedw. v České a Slovenské republice (Musci). - Sborník Západočeského muzea v Plzni, 76 pp.

Authors' addresses: Lukáš Č́íhal, Michaela Mrnková, Silesian Museum, Nádražní okruh 31, CZ-746 01 Opava, Czech Republic, e-mail: cihal@szm.cz; mrnkova.michaela@seznam.cz;

Oto Kaláb, Department of Biology and Ecology, University of Ostrava, Chittussiho 10, CZ- 71000 Ostrava, Czech Republic; e-mail: kalab.oto@gmail.com 